\title{
RAPID COMMUNICATION \\ Expression of cytokine mRNA during immuno-modulation of murine suppressor macrophages
}

\author{
FENG WEI Guo, ZHONG LIANG CHANG* \\ Shanghai Institute of Cell Biology, Chinese Academy of \\ Science, Shanghai 200031, China \\ E-mail: immusig@sunm.shcnc.ac.cn
}

\begin{abstract}
In order to analyze the mechanism of immunomodulation by LPS on murine peritoneal suppressor macrophages, we have, using RNase protection assay, checked the changes of mRNA expression pattern of several cytokine genes during the immuno-modulation. It has been found that, after treating peritoneal suppressor macrophages with LPS, mRNAs of IL-12 p35, IL-12 p40, IL-6 and IFN- $\gamma$ are newly appeared, while those of IL$1 \alpha$, IL-1 $\beta$ and IL-1Ra are increased and those of other cytokines, like TGF- $\beta 1$ and MIF are not changed at all. It seems certain that those cytokines, whose expression is increased by LPS stimulation, may be responsible for the functional changes of suppressor macrophages during immuno-modulation. Among these changes, the appearance of IL-12 mRNA may play a critical role, and, in this regard, the synergetic effect between IFN- $\gamma$ and LPS on the increase of IL-12 p35 and IL-12 p40 mRNA expression is an interesting finding.
\end{abstract}

Key words: Suppressor macrophage, cytokine, $m R N A$ expression, immuno-modulation.

\section{INTRODUCTION}

In our previous experiments, LPS (lipopolysaccharide) was found to be a potent immuno-modulator which could transform the murine peritoneal suppressor

* Corresponding author 
macrophages (MPSMs) into helper ones, whereby they retain the original antitumor activity of suppressor macrophages, but decrease their inhibitory effect on T, B lymphocytes and NK cells in vitro. We called this phenomenon immuno-modulation. It has also been found that helper macrophages secrete more IL-1 and IL-12 proteins and synthesize more cell surface Fc receptors than suppressor macrophages[1, 2].

Cytokines are low molecular-weight, soluble peptides or glycoproteins which can be secreted by many kinds of cells in paracrine or autocrine fashion and play an important role in immuno-regulation, inflammatory reaction, tissue regeneration, cell differentiation, embryo development[3]. Their production is transient and highly controlled. As we all know, macrophages can produce many different cytokines[4]; however, it is interesting and important to know which cytokines are related to the immuno-modulation. Thus, we have used RNase protection assay to investigate the changes of many cytokine mRNAs during the immuno-modulation, including IL-12 p35, IL-12 p40, IL-1 $\alpha$, IL-1 $\beta$, IL-1Ra, IL-6, IFN- $\gamma 1$, MIF, TGF- $\beta 1$ and TNF- $\alpha$, with the finding that the change of IL-12 may be the most attractive one.

IL-12 is a heterodimeric cytokine produced by macrophages in response to intracellular pathogens and bacterial products. Among its many biological activities, IL-12 provides an obligatory signal for the differentiation of T-helper 1 (Th1) effector cells and for the secretion of the Th1 cytokines: gamma interferon (IFN- $\gamma$ ) and IL-2. Th1 cells are required for cell-mediated immunity and host defense against in tracellular microbes. The importance of IL-12 in the generation of a Th1 response against human pathogens as well as during clinical progression of human immunodeficiency virus infection has been well characterized. Further understanding of the regulated expression of IL-12 in macrophages may provide insight into the pathogenesis of infectious and inflammatory diseases and may find novel approaches for modulating immune responses[5].

It has already been reported that binding of LPS and CD14 molecule[6] could result in the translocation of PKC- $a,-\varepsilon$, the activation of Raf- $1 / \mathrm{MAPK} / \mathrm{cPLA}{ }_{2}$ pathway and the increase of the production of cAMP and the activity of PKA[2]. To make clear that which signaling pathway is important in cytokines gene expression, we used forskolin, PMA, IFN- $\gamma$ to activate the PKA, PKC and JAK-STAT pathways respectively, and measured the respective amounts of mRNAs for the various cytokines investigated.

\section{MATERIALS AND METHODS}

\section{Materials}

Animal

Two-month-age mice of C57BL/6J strain weighing 18-20 g were obtained from and maintained in the Animal House of our institute.

Reagents

Mostly obtained from Sigma Company unless otherwise specified. 


\section{Methods}

\section{Preparation of C57BL/6J MPSMs}

Thioglycollate-elicited MPSMs were obtained from the mice injected $4 \mathrm{~d}$ previously with $2 \mathrm{ml}$ of $5 \%$ thioglycollate solution. For details, see references[2].

\section{Treatment of MPSMs}

MPSMs were treated with LPS, PMA or forskolin for $6 \mathrm{~h}$ and harvested for RNA extraction. In order to investigate the effect of IFN- $\gamma$ on the cytokine mRNAs expression in MPSMs, in one set of experiment, we pretreated the MPSMs with IFN- $\gamma$ for $2 \mathrm{~h}$ followed by LPS for another $6 \mathrm{~h}$.

\section{Total RNA extraction}

After MPSMs were homogenized with TRIzol (GIBRL BRL, Cat: 15596-026) and RNA was separated with chloroform, precipitated with isopropyl alcohol, washed with 75\% ethanol, and redissolved in the water treated with $0.1 \%$ DEPC (diethylpyrocarbonate, RNase inhibitor).

\section{RPA (Ribonuclease protection assay)}

RiboQuant MutiProbe RPA system (Pharmingen company, Cat:45024K including $45002 \mathrm{P}$ and $45071 \mathrm{P}$ ) is a highly sensitive method which can quantify many cytokine mRNAs at the same time. In Fig 1 and 2, control RNA (J774.1, LPS, 4h) is supplied by the manufacturer. L32 and GAPDH are all house-keeping genes used as internal standard. L32 is a constitutively expressed gene encoding a ribosomol protein. After hybridization with $\left[a-{ }^{32} \mathrm{P}\right]$ labeled anti-RNA probes (obtained by in vitro transcription according to the RiboQuant protocol)of many cytokines, the total RNA samples were digested by SI RNase which can only hydrolyze the single-chain RNA. Then the labeled double-chain RNA was purified, denatured and separated in 5\% PAGE gel with 8M Urea. At last, the gel was dried, exposed and quantified in a phosphor Imager using the ImageQuant software. Every gene has a corresponding band on the film, and the OD of the band shows the quantity of the corresponding mRNA.

\section{RESULTS AND DISCUSSIONS}

\section{Changes of cytokine mRNAs expression during immuno-modulation}

It was previously reported that LPS could immuno-modulate the MPSMs into helper macrophages with many functional changes. We presumed that it is cytokines secreted by the LPS-modulated macrophages that contribute to the changed phenotype of MPSMs. If so, the LPS-modulated macrophages should secrete some new kinds of cytokines or change the amount of secretion for the already expressed cytokines. Actually, this was found to be true in the present experiment.

Among the cytokines examined, the untreated MPSMs expressed IL- $1 \alpha$, IL-1 $\beta$, IL-1Ra, TGF- $\beta$, MIF and TNF- $\alpha$ mRNAs. When MPSMs were immuno-modulated with LPS, the induction of some new kinds of cytokines mRNAs becomes very evident, such as IL-12 p35, IL-12 p40 (Fig 1), IL-6 and IFN- $\gamma$ (Fig 2), while mRNAs of IL-1 $\alpha$, IL-1 $\beta$ and IL-1Ra are increased (Fig 3) and other cytokines, like TGF- $\beta 1$ and MIF are not changed at all (Fig 4). Those experiments were repeated for 2-3 times with similar results.

From the above-mentioned preliminary results, it is highly probable that those cytokines, such as IL-12 p35, IL-12 p40, IL-6, IFN- $\gamma$ IL-1 $\alpha$, IL-1 $\beta$ and IL-1Ra, 
Cytokine gene expression of LPS-modulated macrophages

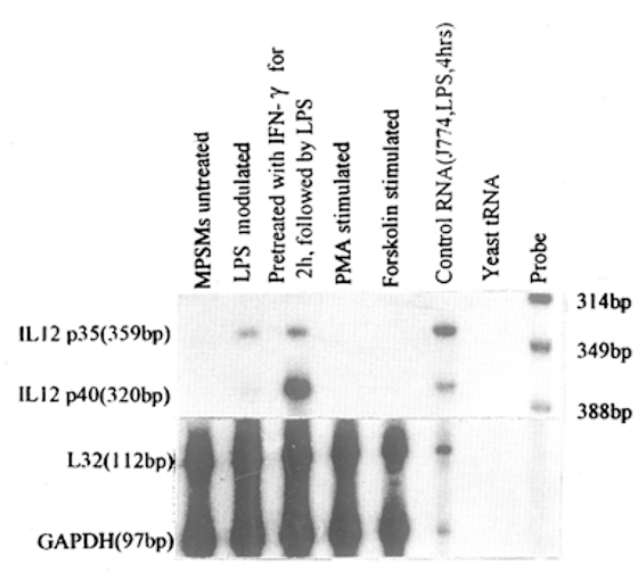

Fig 1. Expression of IL-12 p35 and IL-12 p40 mRNAs in MPSMs.

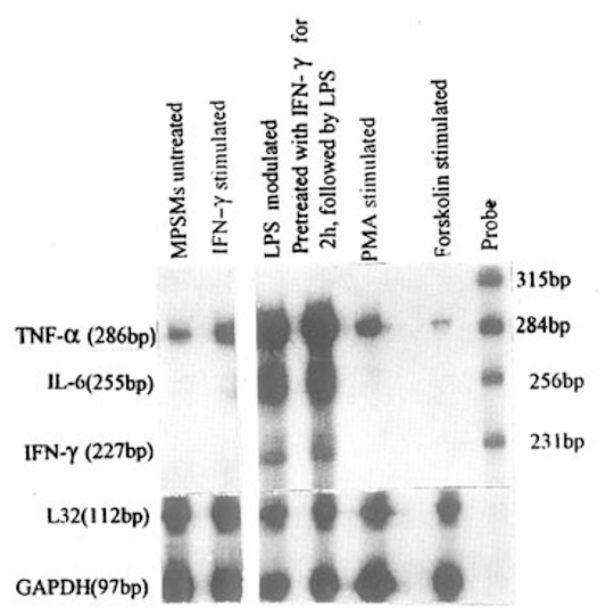

Fig 3. Expression oflL- $1 \alpha, 1-\beta, 1$-Ra mRNAs in MPSMs.

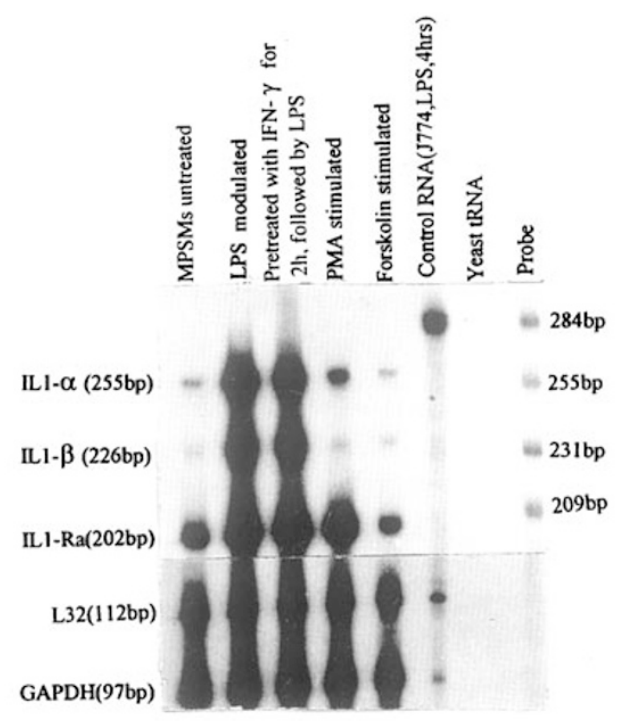

Fig 2. Expression of IL-6, TNF- $a$ and IFN- $\gamma$ mRNAs in MPSMs.

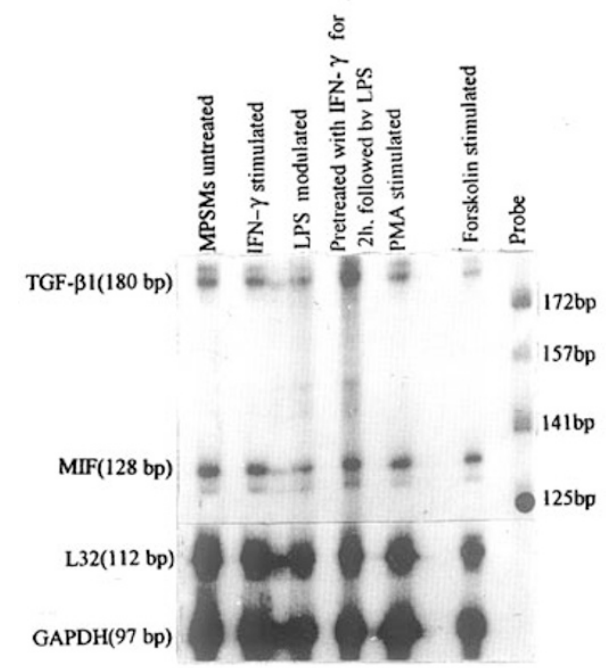

Fig 3. Expression of TGF- $\beta 1$ and MIF mRNAs in MPSMs.

whose expression are increased by LPS stimulation, may be responsible for the functional changes of MPSMs, although their inter-relations and the kinetics of the changes need further studies. 
Effect of IFN- $\gamma$ on the cytokine mRNA expression in the murine peritoneal suppressor macrophages

Turning to the effect of IFN- $\gamma$ on some cytokine mRNA expression in MPSMs, there was no distinct difference between the nonstimulated MPSMs and those stimulated by IFN- $\gamma$ alone (Fig 2, 4). So it is possible that JAK pathway activated specifically by IFN- $\gamma$ may not be directly related to the induction of respective cytokine mRNAs expression in the present experiments. However, it is surprised to find repeatedly that the pretreatment of IFN- $\gamma$ in combination with LPS greatly augmented the expression of IL-12 p40 and IL-12 p35 mRNAs in MPSMs, increased by 16 and 1.6 times respectively as compared with LPS stimulation alone (Fig 1), while IFN- $\gamma$ itself did not stimulate their mRNAs expression (data not shown). In other words, IFN- $\gamma$ and LPS have synergetic effect on IL-12 mRNA expression, although the mechanism for this synergetic phenomenon is not clear yet. Moreover, no such synergetic effect on the mRNA expression of other cytokines can be found in Fig 2-4, indicating that the effect may be rather specific to IL-12.

What is the function of this effect of IFN- $\gamma$ ? IFN- $\gamma$ is produced by activated $\mathrm{CD}^{+}$and $\mathrm{CD}^{+} \mathrm{T}$ cells and by $\mathrm{NK}$ cells. Its transcription is directly resulted as a consequence of antigen activation and is enhanced by IL-2 and IL-12[7]. Considering that IFN- $\gamma$ can enhance IL-12 mRNA expression in macrophages together with LPS, it is suggestive that IFN- $\gamma$ and IL-12 may function as mediators which would connect the macrophages with $\mathrm{T}$ cells between the innate immunity and $\mathrm{T}$ cell-mediated adeptive immunity.

Cell signaling pathways that related to cytokine mRNA expression in peritoneal suppussor macrophages

It has already been reported by us that there are three signal transduction pathways in LPS-activated MPSMs. One is PKC- $a,-\varepsilon$ pathway, the second is Raf$1 / \mathrm{MAPK} / \mathrm{cPLA}$ pathway, and the third is PKA pathway through the increased production of PGE (prostaglandin)[2]. To discern which pathway would be mostly related to the enhanced cytokine gene transcription, PMA, Forskolin were used to activate the different pathways respectively.

The results indicate that PMA did not affect the expression pattern of IL-12 p35, IL-12 p40, IL-6, IFN- $\gamma$ mRNAs (Fig 1, 3) while the expression of IL-1 $\alpha$, IL-1 $\beta$, IL $-1 \mathrm{Ra}$ and TNF- $a$ gene were more or less increased (Fig 2, 3). It is possible that the signaling pathway leading to the expression of IL-12 p35, IL-12 p40, IL-6, and IFN- $\gamma$ mRNAs is different from that for IL- $1 \alpha$, IL- $\beta$, IL-1Ra and TNF- $\alpha$ mRNAs expression, the former depends on PKC pathway while the latter not.

On the other hand, Forskolin, a cAMP inducer, did not show any enhancing effect on cytokine mRNAs expression, and it even showed a slightly inhibitory effect on IL-1Ra, TNF- $\alpha$, TGF- $\beta 1$ and MIF mRNA expression (Fig 2, 3, 4) as compared 
with those of untreated MPSMs. So PKA pathway may be a gating pathway which negatively regulates some cytokine mRNAs expression. Recently it was indirectly demonstrated by Luhm et al that the decrease of cAMP enhanced the LPS-induced cytokine production[9]. It is surprising to note why LPS activates a possibly inhibitory PKA pathway? My be that PKA pathway would have the function to prevent MPSMs from overactivating or overexpressing of cytokine genes.

From above-mentioned considerations, the importance of MAPK pathway in the immuno-modulation effect of LPS is tentatively hinted. This is in agreement with the result of our recent report that LPS can activate Raf-1, MAPK p44 and p42 and cPLA2, with the simultaneous secretion of IL-12 protein[2]. So it is highly probable that MAPK pathway may mediate the LPS-induced cytokine production.

\section{ACKNOWLEDGEMENTS}

We would like to acknowledge to Professor YAO Zhen for his comment and review of the manuscript. We also thank Dr. SUN Hong for her assistance in TGF- $\beta 1$ mRNA determination..

The project was supported by Chinese National Natural Science Fundation (Key Program No. 39630310)

\section{REFERENCES}

[1] Li Xingqiang, Zhang Zong, liang, Yao Zhen. Enhanced expression of Fc receptors after modulation of murine suppressor macrophages by Lipopolysaccharide. Kexue Tongbao, 1988; 33(23):197981.

[2] Zhang Zongliang, Song Qiubao, Lin Mingqun, Ding Yuemei, Kang Xiaowei, Yao Zhen. Immunomodulated signaling in macrophages: studies on activation of Raf-1, MAPK, cPLA and secretion of IL-12. Science in China (series C) 1997; 40(6):583-92.

[3] Jan Vilcek and Junming Le. Immunology of cytokines: an introduction. In: Angus Thomson. Second edition. The Cytokine Handbook. Academic Press Inc: San Diego, CA. 1994:1-19.

[4] Cavaillon JM. Cytokines and Macrophages, Biomed and Pharmacother, 1994; 48:445-53.

[5] Scott E Plevy, James HM, Gemberling et al. Multiple control elements mediate activation of the murine and human interleukin 12 p40 promoters: evidence of functional synergy between C/EBP and Rel proteins, Molecular and Cellular Biology, 1997; 17(8):4572-88.

[6] Thomas A. Hamilton, Yoshihiro Ohmori, Julie M. Tebo, Shosaku Narumi, Charles S. Tannenbaum. Transmembrane and intracellular signaling events in Lipopolysaccharide-stimulated macrophages. In: Bruce S. Zwilling, Toby K. Eisenstein. Marcel Dekker Inc: New York 1994:8397.

[7] Cytokines. In: Abul K. Abbas, Andrew H. Lichtman, Jordan S. Pober. 3rd edition. Cellular and molecular immunology. W.B. Saunders Company: Philadelphia, Pennnsylvania. 1997:249-77.

[8] Luhm J, Kir Chner H, Rink L. One way synergistic effect of low superantigen concentrations on Lipopolysaccharide-induced cytokine production. J Interferon Cytokine Res 1997; 17(4):229-38.

Received Nov-11-1998. Revised Nov-18-1998. Accepted Dec-2-1998. 